\title{
Effect of the radiation component on soliton motion
}

\author{
L. Martínez Alonso \\ Departamento de Métodos Matemáticos de la Física, Facultad de Ciencias Físicas, Universidad Complutense, \\ 28040-Madrid, Spain \\ (Received 11 March 1985)
}

\begin{abstract}
The problem of the soliton motion in the case of a nonzero reflection coefficient is solved exactly within the framework of the inverse scattering method. A general method is given for obtaining the asymptotic expressions of the soliton angle variables. As illustrative examples of our procedure, we consider the Korteweg-de Vries equation and its higher analogs, the nonlinear Schrödinger equation, and the sine-Gordon equation.
\end{abstract}

\section{INTRODUCTION}

In a recent work $^{1}$ we proposed a method for determining the asymptotic positions of the solitons for the case of nonzero reflection coefficient. This procedure which is based on some general properties of the inverse scattering method (ISM) has, however, two limitations. First, it makes use of the expressions of boost generators in terms of scattering data. Although these expressions are available for several of the most relevant completely integrable systems, and not only in the classical context ${ }^{2}$ but also in the quantum one, ${ }^{3}$ there is not yet a proof of their existence for general models solvable by the ISM. On the other hand, the method of Ref. 1 does not apply to finding the asymptotic expressions of the internal variables of the solitons. The present paper aims to show how these difficulties may be overcome through the asymptotic analysis of integral equations of Gel'fand-LevitanMarchenko (GLM) type. In this way we provide here a general method for finding the parameters which characterize the asymptotic trajectories of the solitons arising in the nonlinear models solvable by the ISM. Thus, for these models our results prove that the classical $S$ matrix describing the interaction of a soliton with both the other solitons and the radiation component can be explicitly found.

The asymptotic solution of GLM equations is a difficult mathematical problem which has been considered by numerous authors ${ }^{4-8}$ and from several different points of view. A particularly important progress has been achieved for the solitonless case, but only a few results are known for the general case in which both solitons and radiation component are present. In this paper our analysis is based on the observation that in the ISM the timedependent GLM equation has a peculiar structure which allows us to define a spectrum of velocities associated with the scattering data. It is worth mentioning that for several relevant models ${ }^{2}$ this spectrum may also be found through the interpretation of the normal modes in terms of particles and linear fields associated with the Galilei and Poincare groups. The importance of this spectrum of velocities lies in the fact that it determines the form of the GLM equation in the asymptotic limits $t \rightarrow \pm \infty$. As a consequence, given a solution of an equation solvable by the ISM, we are able to find the scattering data corresponding to the parts of the solution which as $t \rightarrow \pm \infty$ propagate on intervals of the form $\left[x_{0}+\left(v_{0} \pm 0\right) t,+\infty\right)$. Then, by means of a simple argument, without using boost generators, we deduce the explicit expressions of the soliton variables as $t \rightarrow \pm \infty$.

The basic aspects of our analysis are discussed in detail in Sec. II. It deals with the Korteweg-de Vries (KdV) equation and its higher analogs, which can be integrated through the scattering data provided by the Schrödinger spectral problem. We then show how the same scheme can be directly extended to characterize the soliton motion for the nonlinear Schrödinger (NLS) equation and the sine-Gordon (SG) equation in Secs. III and IV, respectively.

\section{KORTEWEG-DE VRIES EQUATION AND ITS HIGHER ANALOGS}

We begin with a brief discussion of the ISM associated with the Schrödinger spectral problem on the line, ${ }^{9}$

$$
\begin{aligned}
& {\left[-\partial_{x x}+u(x)\right] f(k, x)=k^{2} f(k, x),} \\
& f(k, x) \rightarrow e^{i k x}, x \rightarrow+\infty,
\end{aligned}
$$

where $u(x) \rightarrow 0$ as $|x| \rightarrow \infty$. The Jost solution $f(k, x)$ exists for all $k$ in the upper-half plane $\operatorname{lm} k \geq 0$ and determines two scattering-data functions $a(k)$ and $b(k)$ through the asymptotic condition

$f(k, x) \rightarrow a(k) e^{i k x}-b^{*}(k) e^{-i k x}, x \rightarrow-\infty, k \in \mathbb{R}$.

The function $a(k)$, which will be referred to as the transition coefficient of $u(x)$, admits an analytic continuation to the upper-half plane and can be expressed in the form

$$
a(k)=\prod_{n=1}^{N} \frac{k-k_{n}}{k-k_{n}^{*}} \exp \left[\frac{i}{2 \pi} \int_{-\infty}^{\infty} \frac{\ln \left[1-|r(q)|^{2}\right]}{q-k-i 0} d q\right] \text {. }
$$

Here, $r(k)=b(k) / a(k)$ is the reflection coefficient and the numbers $k_{n}$, the zeros of $a(k)$, are purely imaginary. The asymptotic behavior of $f(k, x)$ as $x \rightarrow-\infty$ is described by (2.2) and two further relations 


$$
\begin{aligned}
& f\left(k_{n}, x\right) \rightarrow b_{n} e^{-i k_{n} x}, \quad x \rightarrow-\infty, \\
& f(k, x) \rightarrow a(k) e^{i k x}, \quad x \rightarrow-\infty,
\end{aligned}
$$

$$
\operatorname{Im} k>0, a(k) \neq 0 .
$$

The numbers $b_{n}$ associated with the zeros of $a(k)$ will be called the transition constants of $u(x)$.

In order to characterize the potential, the relevant set of scattering data turns out to be

$$
S=\left\{k_{n}=i \eta_{n}, c_{n}, r(k)\right\}, \quad c_{n}=\left[i b_{n} \dot{a}\left(k_{n}\right)\right]^{-1} .
$$

Thus, if we define the function

$$
\Omega(x)=2 \sum_{n} c_{n} e^{2 i k_{n} x}+\frac{1}{\pi} \int_{-\infty}^{\infty} r(k) e^{2 i k x} d k,
$$

and solve the GLM equation

$$
B(x, y)+\Omega(x+y)+\int_{0}^{\infty} \Omega(x+y+z) B(x, z) d z=0,
$$

then the potential $u(x)$ is given by $u(x)=-\partial_{x} B(x, 0)$. In particular, if we take a set of scattering data $S=\left\{k_{1}=i \eta, c_{1}, r(k) \equiv 0\right\}$ with only one $k_{n}$ and a zero reflection coefficient, the corresponding potential is the soliton

$$
\begin{aligned}
& u_{\mathrm{sol}}(x)=-2 \eta^{2} \operatorname{sech}^{2}[\eta(x-q)], \\
& q=\frac{1}{2 \eta} \ln \frac{c_{1}}{2 \eta}=-\frac{1}{2 \eta} \ln \left|b_{1}\right| .
\end{aligned}
$$

The ISM associated with the Schrödinger spectral problem applies to an infinite family of evolution equations of the form ${ }^{10}$

$$
\partial_{t} u=\partial_{x} R, \quad R=\sum_{m=1}^{M} a_{m} R_{m} .
$$

Here, the $a_{m}$ 's are arbitrary real numbers and the functions $R_{m}$ are determined by means of the recursion relation

$$
\partial_{x} R_{m+1}=\left(-\frac{1}{4} \partial_{x x x}+u \partial_{x}+\frac{1}{2} u_{x}\right) R_{m}, \quad R_{1}=\frac{1}{2} u \text {. }
$$

In particular, for $R=8 R_{2}$ we get the $\mathrm{KdV}$ equation

$$
u_{t}=-u_{x x x}+6 u u_{x}
$$

By means of the ISM one finds that for solutions of Eq. (2.10) the evolution law of the scattering data is

$$
\begin{aligned}
& k_{n}(t)=k_{n}, \quad c_{n}(t)=c_{n} e^{-2 i k_{n} v_{n} t}, \\
& r(t, k)=r(k) e^{-2 i \omega(k) t},
\end{aligned}
$$

where

$$
v_{n}=-\frac{1}{2} \sum_{m=1}^{M} a_{m} k_{m}{ }^{2 m-2}, \omega(k)=-\frac{1}{2} \sum_{m=1}^{M} a_{m} k^{2 m-1}
$$

Consequently, the function (2.7) which characterizes the GLM equation evolves in time according to

$$
\begin{aligned}
\Omega(t, x)= & 2 \sum_{n} c_{n} e^{2 i k_{n}\left(x-v_{n} t\right)} \\
& +\frac{1}{\pi} \int_{-\infty}^{\infty} r(k) e^{2 i[k x-\omega(k) t]} d k .
\end{aligned}
$$

This expression suggests a natural spectrum of velocities associated with the scattering data. Indeed, the terms in the sum propagate with velocities $v_{n}$ and the group velocity of the Fourier modes in the integral term is given by

$$
v_{q}(k)=\dot{\omega}(k) \text {. }
$$

The relevance of this spectrum of velocities can be understood through the asymptotic analysis of the GLM equation. The essential point is to notice that because of the form of Eq. (2.8), the value of the potential $u(t, x)$ at a given point $x_{0}$ depends only on the restriction of $\Omega(t, x)$ to the interval $\left[x_{0},+\infty\right)$. Consequently, in order to determine $u(t, x)$ on a moving interval of the form $\left[x_{0}+\left(v_{0}+0\right) t,+\infty\right)$ as $t \rightarrow+\infty$, the only relevant contributions to $\Omega(t, x)$ arise from those scattering data which propagate with velocity greater than $v_{0}$. This implies that the corresponding GLM equation is characterized by the following expression for $\Omega(t, x)$ (see Appendices $\mathrm{A}$ and B):

$$
\begin{aligned}
\Omega_{+}\left(v_{0} ; t, x\right)= & 2 \sum_{n} \theta\left(v_{n}-v_{0}-0\right) c_{n}(t) e^{2 i k_{n} x} \\
& +\frac{1}{\pi} \int_{-\infty}^{\infty} \theta\left(v_{g}(k)-v_{0}\right) r(t, k) e^{2 i k x},
\end{aligned}
$$

where $\theta$ denotes the step function. Likewise, if we want to determine $u(t, x)$ on intervals of the form $\left[x_{0}+\left(v_{0}-0\right) t,+\infty\right)$ as $t \rightarrow-\infty$, we have to solve the GLM equation with an asymptotic expression for $\Omega(t, x)$ of the form

$$
\begin{aligned}
\Omega_{-}\left(v_{0} ; t, x\right)= & 2 \sum_{n} \theta\left[-\left(v_{n}-v_{0}+0\right)\right] c_{n}(t) e^{2 i k_{0} x} \\
& +\frac{1}{\pi} \int_{-\infty}^{\infty} \theta\left(-\left[v_{g}(k)-v_{0}\right]\right) r(t, k) e^{2 i k x} .
\end{aligned}
$$

The solutions $u_{ \pm}(t, x)$ of the GLM equation corresponding to $\Omega_{ \pm}$represent the contributions to $u(t, x)$ arising from those normal modes of (2.10) which propagate with velocity $v$ such that $\pm\left(v-v_{0}\right)>0$. Furthermore, the forms of $\Omega_{ \pm}$as given by (2.17) and (2.18) mean that in terms of scattering data, $u_{ \pm}(t, x)$ are described by (see Appendix A)

$$
S_{ \pm}\left(v_{0} ; t\right)=\left\{k_{n}, c_{n}(t): \pm\left(v_{n}-v_{0}\right)>0 ; \theta\left( \pm\left[v_{g}(k)-v_{0}\right]\right) r(t, k)\right\}
$$

Observe that as a consequence of (2.3) and (2.19) the transition coefficients of $u_{ \pm}(t, x)$ are 


$$
a_{ \pm}\left(v_{0} ; k\right)=\prod_{n} \cdot \frac{k-k_{n}}{k-k_{n}^{*}} \exp \left[\frac{i}{2 \pi} \int_{-\infty}^{\infty} \theta\left( \pm\left[v_{g}(q)-v_{0}\right]\right) \frac{\ln \left[1-|r(q)|^{2}\right]}{q-k-i 0} d q\right]
$$

where the product extends to those n's such that $\pm\left(v_{n}-v_{0}\right)>0$.

Let us analyze now the soliton motion arising in the long-time asymptotic behavior of a given solution $u(t, x)$ of $(2.10)$. For the reflectionless case $[r(k) \equiv 0]$ it is well known ${ }^{10}$ that as $t \rightarrow \pm \infty$ the solution decomposes into $N$ solitons which move with the velocities $v_{n}$ defined in (2.14). Moreover, one finds that as $t \rightarrow \pm \infty$ the asymptotic trajectories $q_{l}^{ \pm}(t)=q_{l}^{ \pm}+v_{l} t$ of the solitons satisfy ${ }^{10}$

$$
q_{l}^{ \pm}=\frac{1}{2 \eta_{l}} \ln \frac{c_{l}}{2 \eta_{l}}+\frac{1}{\eta_{l}} \sum_{n \neq l} \theta\left( \pm\left(v_{n}-v_{l}\right)\right) \ln \left|\frac{\eta_{l}-\eta_{n}}{\eta_{l}+\eta_{n}}\right| \text {. }
$$

However, in the general case the solution $u(t, x)$ gives rise not only to $N$ solitons with velocities $v_{n}$ but also to a dispersive wave train associated with the degrees of freedom provided by the reflection coefficient. We are now going to see how our asymptotic analysis of the GLM equation allows us to characterize the effect of the dispersive wave train on the soliton motion. To this end let us consider the $l$ th soliton with velocity $v_{l}$ and let $u_{ \pm}(t, x)$ be the parts of the solution $u(t, x)$ which propagate to the right of the soliton as $t \rightarrow \pm \infty$. According to our above considerations, we have that $u_{+}(t, x)$ are described by the sets of scattering data $S_{ \pm}\left(v_{l} ; t\right)$. In the same way, it is natural to assume that

$$
\widetilde{S}_{ \pm}\left(v_{l} ; t\right)=S_{ \pm}\left(v_{l} ; t\right) \cup\left\{k_{l}, c_{l}(t)\right\}
$$

are the sets of scattering data corresponding to the parts $\tilde{u}_{ \pm}(t, x)$ of $u(t, x)$ which result from the addition of the $l$ th soliton to $u_{ \pm}(t, x)$. Indeed, the sets $\widetilde{S}_{ \pm}\left(v_{l} ; t\right)$ represent the normal modes which propagate with velocity $v$ such that $\pm\left(v-v_{l}\right) \geq 0$. Let $\widetilde{a}_{+}(k)$ and $\widetilde{b}_{l}^{ \pm}(t)$ be the transition coefficients and the $l$ th transition constants of $\widetilde{u}_{ \pm}(t, x)$, respectively. Then, from (2.3) and (2.6) it is clear that

$$
\begin{aligned}
\widetilde{a}_{ \pm}(k) & =\frac{k-k_{l}}{k-k_{l}^{*}} a_{ \pm}\left(v_{l} ; k\right), \\
\widetilde{b}_{l}^{ \pm}(t) & =\left[i c_{l}(t) \dot{\tilde{a}}_{ \pm}\left(k_{l}\right)\right]^{-1} \\
& =2 \eta_{l}\left[c_{l}(t) a_{ \pm}\left(v_{l} ; k_{l}\right)\right]^{-1} .
\end{aligned}
$$

Consider now the operation of continuing the Jost solution $f\left(k_{l}, x\right)$ from $+\infty$ to the left of the regions occupied by the potentials $\widetilde{u}_{ \pm}(t, x)$. From the definition (2.4) of the transition constants, after this operation $f\left(k_{l}, x\right)$ will reduce to $\widetilde{b}_{l}^{ \pm}(t) \exp \left(-i k_{l} x\right)$. On the other hand, the same result must be obtained if we first continue $f\left(k_{l}, x\right)$ from $+\infty$ to the right of the $l$ th soliton through the potentials $u_{ \pm}(t, x)$, and then we continue the resulting function to the left of the soliton. From (2.4) and (2.5) we may describe the two steps of the latter process in the form

$$
\begin{aligned}
f\left(k_{l} ; x\right) & \rightarrow a_{ \pm}\left(v_{l} ; k_{l}\right) e^{i k_{l} x} \\
& \rightarrow a_{ \pm}\left(v_{l} ; k_{l}\right) b_{l}^{ \pm}(t) e^{-i k_{l} x},
\end{aligned}
$$

where $b_{l}^{ \pm}(t)$ are the transition constants corresponding to the $l$ th soliton as $t \rightarrow \pm \infty$. It then follows that

$$
\begin{aligned}
b_{l}^{ \pm}(t) & =\widetilde{b}_{l}^{ \pm}(t)\left[a_{ \pm}\left(v_{l} ; k_{l}\right)\right]^{-1} \\
& =2 \eta_{l}\left[c_{l}(t) a_{ \pm}{ }^{2}\left(v_{l} ; k_{l}\right)\right]^{-1}
\end{aligned}
$$

and so, taking into account that

$$
q_{l}^{ \pm}(t)=-\frac{1}{2 \eta_{l}} \ln \left|b_{l}^{ \pm}(t)\right|
$$

we find

$$
q_{l}^{ \pm}=\frac{1}{2 \eta_{l}} \ln \frac{c_{l}}{2 \eta_{l}}+\frac{1}{\eta_{l}} \ln \left|a_{ \pm}\left(v_{l} ; k_{l}\right)\right| .
$$

Hence, by using (2.20) we get

$$
\begin{aligned}
q_{l}^{ \pm}=\frac{1}{2 \eta_{l}} \ln \frac{c_{l}}{2 \eta_{l}} & +\frac{1}{\eta_{l}} \sum_{n \neq l} \theta\left( \pm\left(v_{n}-v_{l}\right)\right) \ln \left|\frac{\eta_{l}-\eta_{n}}{\eta_{l}+\eta_{n}}\right| \\
-\frac{1}{2 \pi} \int_{-\infty}^{\infty} \theta\left( \pm\left(v_{g}(k)-v_{l}\right)\right) & \\
& \times \frac{\ln \left[1-|r(k)|^{2}\right]}{k^{2}+\eta_{l}^{2}} d k
\end{aligned}
$$

The integral term in Eq. (2.27) shows clearly the effect of the dispersive wave train on the soliton trajectories. According to (2.27) the shift in position experienced by the lth soliton due to the presence of both the other solitons and the dispersive wave train is given by

$$
\begin{aligned}
q_{l}^{+}-q_{l}^{-}= & \frac{1}{\eta_{l}} \sum_{n \neq l} \operatorname{sgn}\left(v_{n}-v_{l}\right) \ln \left|\frac{\eta_{l}-\eta_{n}}{\eta_{l}+\eta_{n}}\right| \\
& -\frac{1}{2 \pi} \int_{-\infty}^{\infty} \operatorname{sgn}\left[v_{g}(k)-v_{l}\right] \frac{\ln \left[1-|r(k)|^{2}\right]}{k^{2}+\eta_{l}^{2}}
\end{aligned}
$$

For the case of the $\mathrm{KdV}$ equation $v_{g}(k)=-12 k^{2}$ and $v_{l}=4 \eta_{l}^{2}$ so that $v_{g}(k)-v_{l}$ is strictly negative. As a consequence one may find ${ }^{11}$ that (2.27) and (2.28) correspond to the results obtained by Ablowitz and Kodama. ${ }^{8}$

\section{NONLINEAR SCHRÖDINGER EQUATION}

We shall now apply the method of the last section to the nonlinear Schrödinger (NLS) equation

$$
i \psi_{t}=-\psi_{x x}-2|\psi|^{2} \psi
$$

which can be solved by means of the ISM associated with 
the Zakharov-Shabat spectral problem ${ }^{12}$

$$
\begin{aligned}
& {\left[i \sigma_{3} \partial_{x}+\left[\begin{array}{cc}
0 & \psi(x) \\
-\psi^{*}(x) & 0
\end{array}\right]-k\right] f(k, x)=0,} \\
& f(k, x) \rightarrow\left[\begin{array}{l}
0 \\
1
\end{array}\right] e^{i k x}, \quad x \rightarrow+\infty .
\end{aligned}
$$

We may define scattering data for (3.2) which are completely similar to the ones used in the Schrödinger spectral problem. They are introduced through the relations

$$
\begin{aligned}
& f(k, x) \rightarrow a(k)\left[\begin{array}{l}
0 \\
1
\end{array}\right] e^{i k x}+b^{*}(k)\left[\begin{array}{l}
1 \\
0
\end{array}\right] e^{-i k x}, \\
& x \rightarrow-\infty, k \in \mathbb{R} ; \\
& a(k)=\prod_{n=1}^{N} \frac{k-k_{n}}{k-k_{n}^{*}} \exp \left[\frac{i}{2 \pi} \int_{-\infty}^{\infty} \frac{\ln \left[1+|r(q)|^{2}\right]}{q-k-i 0} d q\right] \\
& f\left(k_{n}, x\right) \rightarrow b_{n}\left[\frac{1}{0}\right] e^{-i k_{n} x}, x \rightarrow-\infty ;(k) \\
& f(k, x) \rightarrow a(k)\left[\begin{array}{l}
0 \\
1
\end{array}\right] e^{i k x}, \\
& x \rightarrow-\infty, \quad \operatorname{Im} k>0, a(k) \neq 0 .
\end{aligned}
$$

The potential $\psi(x)$ in the Zakharov-Shabat spectral problem may be reconstructed from the set of scattering data

$S=\left\{k_{n}=\xi_{n}+i \eta_{n}, c_{n}, r(k)\right\}, \quad c_{n}=\left[i b_{n} \dot{a}\left(k_{n}\right)\right]^{-1}$,

by solving a system of integral equations of GLM type of the form

$B_{1}(x, y)-\Omega^{*}(x+y)-\int_{0}^{\infty} \Omega^{*}(x+y+z) B_{2}^{*}(x, z) d z=0$,

$B_{2}^{*}(x, y)+\int_{0}^{\infty} \Omega(x+y+z) B_{1}(x, z) d z=0$,

$\psi(x)=-i B_{1}(x, 0)$,

where

$$
\Omega(x)=2 \sum_{n} c_{n} e^{2 i k_{n} x}+\frac{1}{\pi} \int_{-\infty}^{\infty} r(k) e^{2 i k x} d k
$$

In particular, the soliton of the NLS equation can be written as

$$
\begin{aligned}
& \psi_{\mathrm{sol}}(x)=-2 \eta \exp [i(\phi-2 \xi x)] \operatorname{sech}[2 \eta(x-q)], \\
& q=-\frac{1}{2 \eta} \ln \left|b_{1}\right|, \quad \phi=\arg b_{1},
\end{aligned}
$$

with

$$
S=\left\{k_{1}=\xi+i \eta, \quad c_{1}=2 \eta b_{1}^{-1}, \quad r(k) \equiv 0\right\}
$$

being its associated set of scattering data.

By means of the Lax-pair technique it is straightforward to find that under the NLS flow the scattering data evolve in time according to

$$
\begin{aligned}
& k_{n}(t)=k_{n}, \quad c_{n}(t)=c_{n} e^{4 i k_{n}{ }^{2} t}, \\
& r(t, k)=r(k) e^{4 i k^{2} t} .
\end{aligned}
$$

Hence, the time evolution of the function (3.9) is given by

$$
\begin{aligned}
\Omega(t, x)= & 2 \sum_{n} c_{n} e^{2 i\left(k_{n} x+2 k_{n}^{2} t\right)} \\
& +\frac{1}{\pi} \int_{-\infty}^{\infty} r(k) e^{2 i\left(k x+2 k^{2} t\right)} d k .
\end{aligned}
$$

Observe that the modulus of the $n$th term in the sum propagates with velocity $v_{n}=-4 \xi_{n}$, while the group velocity of the Fourier modes in the integral term is $v_{g}(k)=-4 k$. It must be noticed that, as in the Schrödinger spectral problem, because of the form of the GLM equations (3.8) the value of $\psi(t, x)$ at a given point $x_{0}$ depends only on the restriction of $\Omega(t, x)$ on the interval $\left[x_{0}+\infty\right)$; therefore, we may perform the same asymptotic analysis as in Sec. II. The result is that as $t \rightarrow \pm \infty$ the transition constants [see (3.5)] corresponding to the $l$ th soliton of the NLS field are given by

$$
b_{l}^{ \pm}(t)=2 \eta_{l}\left[c_{l}(t) a_{ \pm}{ }^{2}\left(v_{l} ; k_{l}\right)\right]^{-1} .
$$

Here, the functions $a_{ \pm}$are the transition coefficients corresponding to the parts of the NLS field which propagate to the right of the $l$ th soliton as $t \rightarrow \pm \infty$. Thus, taking into account (3.10), it follows that the asymptotic expressions for the angle variables of the solitons are

$$
\begin{aligned}
& q_{l}^{ \pm}= \frac{1}{2 \eta_{l}} \ln \frac{\left|c_{l}\right|}{2 \eta_{l}}+\frac{1}{\eta_{l}} \sum_{n \neq l} \theta\left( \pm\left(v_{n}-v_{l}\right)\right) \ln \left|\frac{k_{l}-k_{n}}{k_{l}-k_{n}^{*}}\right| \\
&-\frac{1}{2 \pi} \int_{-\infty}^{\infty} \theta\left( \pm\left[v_{g}(k)-v_{l}\right]\right) \\
& \times \frac{\ln \left[1+|r(k)|^{2}\right]}{\left(k-\xi_{l}\right)^{2}+\eta_{l}^{2}} d k \\
&\left.\varphi_{l}^{ \pm}=-\arg c_{l}-2 \sum_{n \neq l} \theta\left( \pm\left(v_{n}-v_{l}\right)\right) \arg \mid \frac{k_{l}-k_{n}}{k_{l}-k_{n}^{*}}\right) \\
&-\frac{1}{\pi} \int_{-\infty}^{\infty} \theta\left( \pm\left[v_{g}(k)-v_{l}\right]\right) \\
& \times \frac{\left(k-\xi_{l}\right) \ln \left[1+|r(k)|^{2}\right]}{\left(k-\xi_{l}\right)^{2}+\eta_{l}^{2}} d k
\end{aligned}
$$

\section{SINE-GORDON EQUATION}

The analysis of the soliton motion for the sine-Gordon equation

$$
\phi_{t t}-\phi_{x x}+\sin \phi=0
$$

may be carried out by following the same scheme as in Secs. II and III. For this model, the auxiliary spectral problem is provided by ${ }^{13}$

$$
\left[J \partial_{x}+A+\frac{1}{k} B-k\right] f(k, x)=0,
$$




$$
f(k, x) \rightarrow e(k, x)=\left(\begin{array}{l}
1 \\
i
\end{array}\right) \exp \left[i\left(k-\frac{1}{16 k}\right) x\right]
$$$$
x \rightarrow+\infty \text {, }
$$

where

$$
\begin{aligned}
& J=\left(\begin{array}{cc}
0 & -1 \\
1 & 0
\end{array}\right], \quad A=\frac{i}{4}\left(\begin{array}{cc}
0 & \phi_{x}+\phi_{t} \\
\phi_{x}+\phi_{t} & 0
\end{array}\right), \\
& B=\frac{1}{16}\left(\begin{array}{cc}
e^{i \phi} & 0 \\
0 & e^{-i \phi}
\end{array}\right):
\end{aligned}
$$

The scattering data may be defined in the same fashion as for the Schrödinger and Zakharov-Shabat spectral problems. Thus, we have the relations

$$
\begin{gathered}
f(k, x) \rightarrow a(k) e(k, x)+b^{*}(k) e^{*}(k, x), \\
x \rightarrow-\infty, k \in \mathbb{R} ; \\
a(k)=\prod_{n=1}^{N} \frac{k-k_{n}}{k-k_{n}^{*}} \exp \left[\frac{i}{2 \pi} \int_{-\infty}^{\infty} \frac{\ln \left(1+|r(q)|^{2}\right)}{q-k-i 0} d q\right], \\
f\left(k_{n}, x\right) \rightarrow b_{n}\left[\begin{array}{c}
1 \\
-i
\end{array}\right] \exp \left[-i\left[k_{n}-\frac{1}{16 k_{n}}\right] x\right], x \rightarrow-\infty ;
\end{gathered}
$$

$f(k, x) \rightarrow a(k) e(k, x), \quad x \rightarrow \infty, \quad \operatorname{Im} k>0, \quad a(k) \neq 0$.

In terms of scattering data the SG field is determined from the set

$$
S=\left\{k_{n}=\frac{1}{4} e^{-\beta_{n}+i \theta n}, c_{n}, r(k)\right\}, c_{n}=\left[i b_{n} \dot{a}\left(k_{n}\right)\right]^{-1} .
$$

By means of the resolution of a system of integral equations of GLM type given by

$$
\begin{array}{r}
B_{1}(x, y)+\Omega_{1}(x+y)+\int_{0}^{\infty} \Omega_{1}(x+y+z) B_{1}(x, z) d z \\
+\int_{0}^{\infty} \Omega_{2}(x+y+z) B_{2}(x, z) d z=0 \\
16 B_{2}(x, y)-\Omega_{2}(x+y)-\int_{0}^{\infty} \Omega_{2}(x+y+z) B_{1}(x, z) d z \\
+\int_{0}^{\infty} \Omega_{3}(x+y+z) B_{2}(x, z) d z=0, \\
\phi(x)=-i \ln \left\{\left[1+8 i B_{2}(x, 0)\right]\left[1-8 i B_{2}(x, 0)\right]^{-1}\right\}
\end{array}
$$

where

$$
\begin{aligned}
& \Omega_{j}(x)= 2 \sum_{n} k_{n}{ }^{1-j} c_{n} \exp \left[2 i\left(k_{n}-\frac{1}{16 k_{n}}\right] x\right] \\
&+\frac{1}{\pi} \int_{-\infty}^{\infty} k^{1-j_{r}}(k) \exp \left[2 i\left(k-\frac{1}{16 k}\right) x\right] d k \\
& j=1,2,3
\end{aligned}
$$

A particular property of the spectral problem (4.2) is that the zeros $k_{n}$ of $a(k)$, as well as the transition constants $b_{n}$, turn out to be located symmetrically relative to the imaginary axis. Owing to this fact, there are two kinds of solitons, namely, kinks

$$
\begin{aligned}
& \phi_{k}(x)=4 \tan ^{-1}\{\exp [ \pm \cosh \beta(x-q)]\}, \\
& S=\left\{k_{1}=\frac{1}{4} e^{-\beta+i \pi / 2}, \quad c_{1}=2\left|k_{1}\right| b_{1}{ }^{-1}, \quad r(k) \equiv 0\right\},
\end{aligned}
$$

$$
q=-(\cosh \beta)^{-1} \ln \left|b_{1}\right|
$$

and breathers

$$
\begin{aligned}
& \phi_{b}(x)=4 \tan ^{-1}\left[\tan \theta \frac{\sin (\varphi-x \sinh \beta)}{\cos [\sin \theta \cosh \beta(x-q)]}\right], \\
& S=\left\{k_{1}=-k_{2}^{*}=\frac{1}{4} e^{-\beta+i \theta}, c_{1}=-c_{2}^{*}=2 \operatorname{Im} k_{1} b_{1}^{-1}, r(k) \equiv 0\right\}, \\
& q=-(\sin \theta \cosh \beta)^{-1} \ln \left|b_{1}\right|, \varphi=-2 \arg \left(-i b_{1}\right) .
\end{aligned}
$$

Under the evolution law generated by the SG equation the zeros $k_{n}$ of $a(k)$ are independent of time, while

$$
c_{n}(t)=c_{n} \exp \left[2 i\left[k_{n}+\frac{1}{16 k_{n}}\right) t\right], r(t, k)=r(k) \exp \left[2 i\left(k+\frac{1}{16 k}\right) t\right] \text {. }
$$

Thus, by inserting these expressions into Eq. (4.10) for $\Omega_{j}(x)$, it follows that the spectrum of velocities associated with the scattering data is given by

$$
v_{n}=\frac{1-16\left|k_{n}\right|^{2}}{1+16\left|k_{n}\right|^{2}}, \quad v_{g}(k)=\frac{1-16 k^{2}}{1+16 k^{2}} .
$$

At this point and owing again to the form of the GLM equations, one may perform the same procedure for characterizing the soliton trajectories as in the above sections. Consequently, taking into account (4.11) and (4.12), it follows that for both kinks and breathers we have 


$$
\begin{aligned}
q_{l}^{ \pm}= & \left(\sin \theta_{l} \cosh \beta_{l}\right)^{-1}\left[\ln \frac{\left|c_{l}\right|}{2 \operatorname{Im} k_{l}}+2 \sum_{n \neq l} \theta\left( \pm\left(v_{n}-v_{l}\right)\right) \ln \left|\frac{k_{l}-k_{n}}{k_{l}-k_{n}^{*}}\right|\right) \\
& -\left[2 \pi\left(1+e^{2 \beta_{l}}\right)\right]^{-1} \int_{-\infty}^{\infty} \theta\left( \pm\left(v_{g}(k)-v_{l}\right)\right) \frac{\ln \left[1+|r(k)|^{2}\right]}{\left|k-k_{l}\right|^{2}} d k
\end{aligned}
$$

where for kinks the value $\theta_{l}=\pi / 2$ is assumed. In addition, it is found that the breathers satisfy

$$
\begin{aligned}
\varphi_{\bar{l}}^{ \pm}= & 2 \arg \left(-i c_{l}\right)+4 \sum_{n \neq l} \theta\left( \pm\left(v_{n}-v_{l}\right)\right) \arg \left(\frac{k_{l}-k_{n}}{k_{l}-k_{n}^{*}}\right) \\
& +\frac{2}{\pi} \int_{-\infty}^{\infty} \theta\left( \pm\left(v_{g}(k)-v_{l}\right)\right) \frac{\left(k-\operatorname{Re} k_{l}\right) \ln \left[1+|r(k)|^{2}\right]}{\left|k-k_{l}\right|^{2}} d k
\end{aligned}
$$

\section{ACKNOWLEDGMENTS}

This work was financially supported by the Comision Asesora de Investigación Cientifica y Técnica.

\section{APPENDIX A}

In this appendix we outline an asymptotic analysis of integral equations of GLM type which justifies most of the arguments used in the present paper. Although we will consider only the Schrödinger spectral problem, the strategy used applies easily to other cases. The starting point is the fact that the GLM equation (2.8) is equivalent to the following integral equation for the Jost solution (2.1):

$$
\begin{aligned}
f(k, x)= & e^{i k x}-i \sum_{n} \frac{e^{i\left(k+k_{n}\right) x}}{k+k_{n}} c_{n} f\left(k_{n}, x\right) \\
& +\frac{1}{2 \pi i} \int_{-\infty}^{\infty} \frac{e^{i(k+q) x}}{k+q+i 0} r(q) f(q, x) d q,
\end{aligned}
$$

in which the variable $x$ plays the role of a parameter. For a fixed value of $x$, the unknowns in (A1) are the function $f(k, x)(k \in \mathbb{R})$ and the numbers $f\left(k_{n}, x\right)(n=1, \ldots, N)$. Given a solution $u(t, x)$ of Eq. (2.10) its corresponding Jost solution $f(t, k, x)$ will satisfy Eq. (A1) with the scattering data (2.13). Let us consider the asymptotic solution of (A1) on the lines $x=x_{0}+\left(v_{0}+0\right) t$ as $t \rightarrow+\infty$. We look for a solution of the form

$$
\begin{aligned}
& f(t, k, x)=\widetilde{a}(k) e^{i k x}-\widetilde{b}(-k) e^{-i k x+2 i \omega(k) t}, \quad k \in R \\
& f\left(t, k_{n}, x\right)=\widetilde{a}\left(k_{n}\right) e^{i k_{n} x}, \quad v_{n} \leq v_{0} ; \\
& f\left(t, k_{n}, x\right)=\widetilde{b}_{n} e^{-i k_{n} x+2 i k_{n} v_{n} t}, \quad v_{n}>v_{0}
\end{aligned}
$$

On the basis of the properties of the Schrödinger spectral problem, we also assume that

$$
\widetilde{a}^{*}(k)=\widetilde{a}(-k), \quad \widetilde{b}^{*}(k)=\widetilde{b}(-k),
$$

$$
|\widetilde{a}(k)|^{2}-|\widetilde{b}(k)|^{2}=1, \quad k \in \mathbb{R},
$$

and that $\widetilde{a}(k)$ admits an analytic continuation to the upper-half plane. Inserting (A2)-(A4) into (A1) and using the relation ${ }^{6}$

$$
\begin{aligned}
& \int_{-\infty}^{\infty} \frac{e^{i \varphi(q) t}}{q+k+i 0} g(q) d g \rightarrow-2 \pi i \theta(-\dot{\varphi}(-k)) \\
& \times e^{i \varphi(-k) t} g(-k) \\
& t \rightarrow+\infty, k \in \mathbb{R},
\end{aligned}
$$

we get that for real $k \mathrm{Eq}$. (A1) reduces to the following pair of equations:

$$
\begin{aligned}
\widetilde{a}(k)= & 1-i \sum_{n} \theta\left(v_{n}-v_{0}-0\right) \frac{\widetilde{b}_{n} c_{n}}{k+k_{n}} \\
& -\frac{1}{2 \pi i} \int_{-\infty}^{\infty} \frac{r(q) \widetilde{b}^{*}(q)}{q+k+i 0} d q, \\
\widetilde{b}(k)= & \theta\left(v_{g}(k)-v_{0}\right) r(k) \widetilde{a}(k) .
\end{aligned}
$$

For $k=k_{l}$ such that $v_{l} \leq v_{0}$, Eq. (A1) takes the form (A7) with $k=k_{l}$; while for those $k_{l}$ such that $v>v_{0}$, Eq. (A1) is equivalent to

$$
\begin{aligned}
1-i \sum_{n} \theta\left(v_{n}-v_{0}-0\right) & \frac{\widetilde{b}_{n} c_{n}}{k_{l}+k_{n}} \\
& -\frac{1}{2 \pi i} \int_{-\infty}^{\infty} \frac{r(q) \widetilde{b}^{*}(q)}{q+k_{l}} d q=0 .
\end{aligned}
$$

Now, it is not difficult to check that these equations are satisfied if we take [see Eq. (2.20)]

$$
\begin{aligned}
& \widetilde{a}(k)=a_{+}\left(v_{0} ; k\right), \\
& \widetilde{b}(k)=\theta\left(v_{g}(k)-v_{0}\right) r(k) a_{+}\left(v_{0} ; k\right), \\
& \widetilde{b}_{n}=\left[i c_{n} \dot{a}_{+}\left(v_{0} ; k_{n}\right)\right]^{-1} .
\end{aligned}
$$

In particular, observe that from (A5) and (A10) one deduces

$$
\begin{aligned}
-\frac{1}{2 \pi i} \int_{-\infty}^{\infty} \frac{r(q) \widetilde{b}^{*}(q)}{q+k+i 0} d q & =\frac{1}{2 \pi i} \int_{-\infty}^{\infty} \frac{1-\widetilde{a}(-q)}{q+k+i 0} d q+\frac{1}{2 \pi i} \int_{-\infty}^{\infty} \frac{\widetilde{a}(q)^{-1}-1}{q+k+i 0} d q \\
& =\widetilde{a}(k)-1+\sum_{n} \theta\left(v_{n}-v_{0}-0\right)\left[\dot{\widetilde{a}}\left(k_{n}\right)\left(k_{n}+k\right)\right]^{-1}, \quad \operatorname{Im} k>0
\end{aligned}
$$


The form of the asymptotic solution (A2)-(A4) determined by (A10) shows that as $t \rightarrow+\infty$ the restriction of $u(t, x)$ on a moving interval $\left[x_{0}+\left(v_{0}+0\right) t,+\infty\right)$ is described by the set of scattering data $S_{+}\left(v_{0} ; t\right)$ of Eq. (2.19). The analysis of the $t \rightarrow-\infty$ limit may be performed in a completely analogous way.

\section{APPENDIX B}

The functions $\Omega$ which characterize the integral equations of GLM type have a common structure: a finite sum of contributions arising from the discrete scattering data, and an integral term which is the Fourier transform of the reflection coefficient. This property together with the evolution law of the scattering data is used in the present paper to state certain asymptotic expressions $\Omega_{ \pm}$ for $\Omega$ as $t \rightarrow \pm \infty$. In this appendix we provide a precise formulation of the way in which $\Omega$ reduces to $\Omega_{ \pm}$asymptotically. Thus, we will show that under mild conditions, for all $\epsilon>0$ it is verified that $\lim _{t \rightarrow \pm \infty} \int_{x_{0}+\left(v_{0} \pm \in\right) t}^{+\infty}\left|\Omega(t, x)-\Omega_{ \pm}\left(v_{0} ; t, x\right)\right|^{2} d x=0$,

where $x_{0}$ and $v_{0}$ are arbitrary numbers. That is to say, the $L^{2}$ norm of $\Omega-\Omega_{ \pm}$on intervals of the form $\left[x_{0}+\left(v_{0} \pm \epsilon\right) t,+\infty\right)$ vanishes as $t \rightarrow \pm \infty$.

It is easy to see that the contributions of the discrete scattering data to $\Omega-\Omega_{ \pm}$have $L^{2}$ norms on $\left[x_{0}+\left(v_{0} \pm \epsilon\right) t,+\infty\right)$ which tend to zero exponentially as $t \rightarrow \pm \infty$. Thus, in order to prove (A1) we will consider only the integral term of $\Omega-\Omega_{ \pm}$. In the case of $\Omega-\Omega_{+}$ that term is of the form

$$
G(t, x)=\int_{-\infty}^{\infty} \theta\left(v_{0}-v_{g}(k)\right) g(k) e^{i[k x-\omega(k) t]} d k,
$$

where the function $g(k)$, which represents the reflection coefficient, vanishes asymptotically as $|k| \rightarrow \infty$. We first prove that there exists a constant $C_{1}$ independent of $t$ verifying

$$
|t G(t, x)|<C_{1}, \text { for } x \geq\left(v_{0}+\epsilon\right) t .
$$

To this end we perform the following integration by parts,

$$
\begin{aligned}
G(t, x)= & \int_{-\infty}^{\infty} \theta\left(v_{0}-v_{g}(k)\right) \frac{g(k)}{i\left[x-v_{g}(k) t\right]} \partial_{k} e^{i[k x-\omega(k) t]} d k \\
= & -i \sum_{j} \frac{g\left(k_{j}\right)}{x-v_{0} t} e^{i\left[k_{j} x-\omega\left(k_{j}\right) t\right]} \\
& +i \int_{-\infty}^{\infty} \theta\left(v_{0}-v_{g}(k)\right)\left(\frac{\dot{g}(k)}{x-v_{g}(k) t}+\frac{g(k) \dot{v}_{g}(k) t}{\left[x-v_{g}(k) t\right]^{2}}\right) e^{i[k x-\omega(k) t]} d k,
\end{aligned}
$$

where $k_{j}$ denotes the points for which $v_{g}\left(k_{j}\right)=v_{0}$. Now, from (B4) it follows that for all $x \geq\left(v_{0}+\epsilon\right) t$

$$
\left.|t G(t, x)| \leq \sum_{j} \frac{1}{\epsilon}\left|g\left(k_{j}\right)\right|+\int_{-\infty}^{\infty} \theta\left(v_{0}-v_{g}(k)\right) \frac{1}{\epsilon}|| \dot{g}(k)\left|+\frac{1}{\epsilon}\right| g(k) \dot{v}_{g}(k) \mid\right) d k .
$$

This proves (B3). The next step is to show that there exists a constant $C_{2}$ independent of $t$ such that

$$
|x G(t, x)|<C_{2} \text {, for } x \geq\left(v_{0}+\epsilon\right) t
$$

The proof is based on the following integration by parts:

$$
\begin{aligned}
x G(t, x)= & -i \int_{-\infty}^{\infty} \theta\left(v_{0}-v_{g}(k)\right) g(k) e^{-i \omega(k) t} \partial_{k} e^{i k x} d k \\
= & -i \sum_{j} g\left(k_{j}\right) e^{i\left[k_{j} x-\omega\left(k_{j}\right) t\right]}+i \int_{-\infty}^{\infty} \theta\left(v_{0}-v_{g}(k)\right) \dot{g}(k) e^{i[k x-\omega(k) t)]} d k \\
& +t \int_{-\infty}^{\infty} \theta\left(v_{0}-v_{g}(k)\right) g(k) v_{g}(k) e^{i[k x-\omega(k) t]} d k
\end{aligned}
$$

Observe that provided $g(k) v_{g}(k)$ vanishes asymptotically, the coefficient of $t$ in (B6) is an integral of the form (B2), so from (B3) there exists a constant $C_{1}$ such that for all $x \geq\left(v_{0}+\epsilon\right) t$ we have

$$
\begin{aligned}
|x G(t, x)| \leq & \sum_{j}\left|g\left(k_{j}\right)\right| \\
& +\int_{-\infty}^{\infty} \theta\left(v_{0}-v_{g}(k)\right)|\dot{g}(k)| d k+C_{1},
\end{aligned}
$$

and therefore (B5) holds. Clearly (B5) implies that

$$
\lim _{t \rightarrow+\infty} \int_{x_{0}+\left(v_{0}+\epsilon\right) t}^{\infty}|G(t, x)|^{2} d x=0
$$

which is what we wanted to demonstrate. This proves the $t \rightarrow+\infty$ part of (B1); the proof of the $t \rightarrow-\infty$ part may be carried out in the same manner. 
${ }^{1}$ L. Martinez Alonso, Phys. Rev. Lett. 54, 499 (1985).

${ }^{2}$ L. Martinez Alonso, J. Math. Phys. 23, 1518 (1982); 24, 982 (1983); 24, 2652 (1983); Phys. Rev. D 30, 2595 (1984).

${ }^{3}$ M. Göckeler, Z. Phys. C 7, 263 (1981); 11, 125 (1981); K. Sogo and M. Wadati, Prog. Theor. Phys. 69, 431 (1983).

${ }^{4}$ V. E. Zakharov, Zh. Eksp. Teor. Fiz. 60, 993 (1971) [Sov. Phys. JETP 33, 538 (1971)]; M. J. Ablowitz and A. C. Newell, J. Math. Phys. 14, 1277 (1973); H. Segur, J. Fluid Mech. 59, 721 (1973); M. J. Ablowitz and H. Segur, Stud. Appl. Math. 66, 159 (1982).

${ }^{5}$ S. Tanaka, Publ. Res. Inst. Math. Sci. (Kyoto Univ.) 10, 367 (1975).

${ }^{6}$ S. V. Manakov, Zh. Eksp. Teor. Fiz. 65, 1392 (1973) [Sov. Phys. JETP 38, 693 (1974)].

${ }^{7}$ V. E. Zakharov and S. V. Manakov, Zh. Eksp. Teor. Fiz. 71, 203 (1976) [Sov. Phys. JETP 44, 106 (1976)]; V. Ju Novok- senov, Dokl. Akad. Nauk SSSR 251, 799 (1980) [Sov. Math. Dokl. 21, 529 (1980)]; A. R. Its, Dokl. Akad. Nauk SSSR 261, 14 (1981) [Sov. Math. Dokl. 24, 452 (1981)].

${ }^{8}$ M. J. Ablowitz and Y. Kodama, Stud. Appl. Math. 66, 159 (1982).

${ }^{9}$ L. D. Faddeev, Trudy Mat. Inst. Steklov 73, 314 (1964) [Amer. Math. Soc. Transl. 2, 65, 139 (1967)]; P. Deift and E. Trubowitz, Comm. Pure Appl. Math. 32, 121 (1979).

${ }^{10}$ C. S. Gardner, J. M. Green, M. D. Kruskal, and R. Miura, Comm. Pure Appl. Math. 27, 97 (1974).

${ }^{11}$ L. Martinez Alonso, J. Phys. A 17, 2729 (1984).

${ }^{12}$ V. E. Zakharov and A. B. Shabat, Zh. Eksp. Teor. Fiz. 61, 118 (1971) [Sov. Phys. JETP 34, 62 (1972)].

${ }^{13}$ V. E. Zakharov, L. A. Takhtadzhyan, and L. D. Fadeev, Dokl. Akad. Nauk. SSSR 219, 1334 (1974) [Sov. Phys. Dokl. 19, $824(1975)]$. 